\title{
spFRET Microscopy Analysis of Distances Between DNA Linkers in Mononucleosomes
}

Grigoriy A. Armeev ${ }^{1}$, Alexander V. Lubitelev ${ }^{1}$, Vasily M. Studitsky ${ }^{1,3}$, Alexey V. Feofanov ${ }^{1,2}$ and Mikhail P. Kirpichnikov ${ }^{1,2}$

1. Faculty of Biology, Lomonosov Moscow State University, Moscow, Russia.

2. Shemyakin-Ovchinnikov Institute of Bioorganic Chemistry, Russian Academy of Sciences, Moscow, Russia.

3. Fox Chase Cancer Center, Philadelphia, USA.

In cell nuclei DNA is stored in a compact form called chromatin. Numerous structural and regulatory proteins govern structural rearrangements of chromatin and its main structural units, nucleosomes, in order to provide gene expression regulation and DNA replication. Nucleosome linker region plays an important role in chromatin compaction, and the structural organization of this region requires detailed investigation. Using a single-particle Forster resonance energy transfer (spFRET) microscopy we revealed [1] that linker DNA of mononucleosomes adopts two conformations: open (low FRET) and close (high FRET, Figure). Formation of a close conformation by highly charged linker DNA is nontrivial. It is found to be additionally modulated by potassium and sodium ions (Figure).

FRET effect can be used as a molecular ruler to estimate distances between DNA linkers, but to do that the qualitative "proximity ratio" analysis (based on donor and acceptor fluorescence intensity measurements [1]) should be developed to a FRET efficiency analysis. In this way, the detection factor, which accounts for differences between the donor and acceptor detection efficiencies and fluorescence quantum yields of the dyes, has to be measured.

To determine the fluorescence quantum yields of $\mathrm{Cy} 3$ and $\mathrm{Cy} 5$ dyes attached to linker DNA (Figure), we used reference dyes with known fluorescence quantum yields, i.e. rhodamine $6 \mathrm{G}$ in ethanol and ATTO 655 in phosphate buffered saline. Following the approach described elsewhere [2], the fluorescence quantum yields of $\mathrm{Cy} 3$ and $\mathrm{Cy} 5$ dyes conjugated with ssDNA oligonucleotides were measured to be 0.33 and 0.51 , respectively. These values are in a good agreement with data reported for these dyes previously [3]. The ratio of donor and acceptor detection efficiencies was determined by us as proposed elsewhere [4] The resulting detection factor was calculated to be 0.83 . Förster radius was estimated according to the procedure described in [4]. For Cy3 and Cy5 pair of labels attached to a dsDNA oligonucleotide, the Förster radius was measured to be $59.9 \AA$.

In the studied mononucleosomes, Cy3 label was located at a distance of 18 bp before the first nucleotide, and Cy5 was located at a distance of $20 \mathrm{bp}$ after the last nucleotide of the nucleosome positioning sequence. spFRET microscopy measurements of freely diffusing mononucleosomes were performed with the Confocor3 module of the confocal microscope LSM710 (Zeiss, Germany) using Capochromat 40x/1.2 water immersion objective. Fluorescence was excited with the $514 \mathrm{~nm}$ wavelength of $\mathrm{Ar}^{+}$-laser. A combination of optical filters used for spFRET measurements is described elsewhere [5]. Cy3 and $\mathrm{Cy} 5$ intensities measured from single nucleosomes were recalculated into FRET efficiencies and presented as frequency distributions (Figure). On the basis of the measured Förster radius, we estimated that a distance between DNA linkers in the place of label location is equal to $61 \AA$ 
(the close conformation in the presence of potassium ions). This distance increases to $66.4 \AA$, when potassium ions are substituted by sodium ions.

The open conformation of nucleosomes can not be characterized with a distance between DNA linkers on the basis of spFRET microscopy measurements, because FRET efficiency is close to zero for this conformation.

We suppose that changing positions of labels on DNA linkers and measuring interlabel distances, the structure of DNA linker region of mononucleosomes can be probed, and a structural model can be designed using molecular modeling approaches [6].

\section{References:}

[1] Lyubitelev AV et al, Moscow Univ Biol Sci Bull 72 (2017), p. 146.

[2] Rhys Williams AT, Winfield SA and Miller JN, Analyst 108 (1983), p. 1067.

[3] McCann JJ et al, Biophys J. 99 (2010), p. 961.

[4] Ferreon ACM et al, Proc Natl Acad Sci U S A 106 (2009), p. 5645.

[5] Kudryashova KS et al, Methods Mol Biol. 1288 (2015), p. 395.

[6] The authors acknowledge funding by the Russian Science Foundation, grant 14-24-00031.
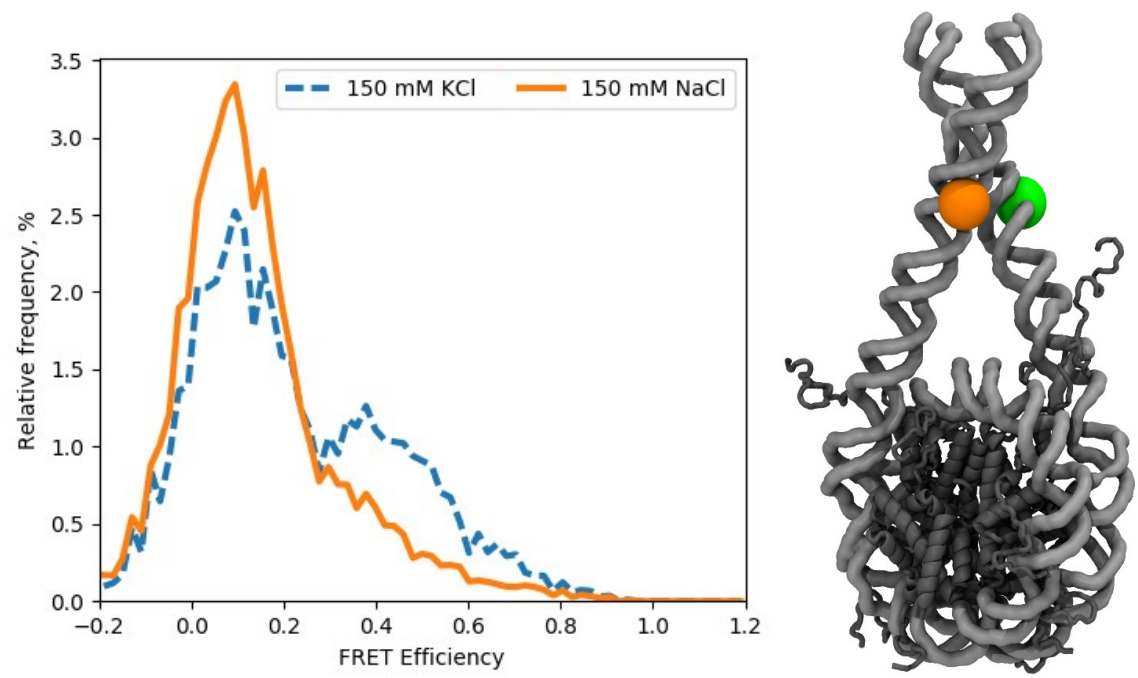

Figure: Left - typical frequency distributions of FRET efficiencies for single nucleosomes in a buffer containing either $150 \mathrm{mM} \mathrm{KCl}$ or $150 \mathrm{mM} \mathrm{NaCl}$ as measured with spFRET microscopy. Right visualisation of a nucleosome with $\mathrm{Cy} 3$ (green) and $\mathrm{Cy} 5$ (orange) fluorescent labels attached to linkers. 